This item was submitted to Loughborough's Research Repository by the author.

Items in Figshare are protected by copyright, with all rights reserved, unless otherwise indicated.

\title{
Developing incident causation constructs for managing safety in construction
}

PLEASE CITE THE PUBLISHED VERSION

http://dx.doi.org/10.3850/978-981-08-7920-4_CS-11-0334

\section{PUBLISHER}

(C) Research Publishing Services

\section{VERSION}

AM (Accepted Manuscript)

\section{LICENCE}

CC BY-NC-ND 4.0

\section{REPOSITORY RECORD}

Al-Shehri, Yousef, Francis Tekyi Edum-Fotwe, and Andrew D.F. Price. 2019. "Developing Incident Causation Constructs for Managing Safety in Construction". figshare. https://hdl.handle.net/2134/14374. 
This item was submitted to Loughborough's Institutional Repository (https://dspace.lboro.ac.uk/) by the author and is made available under the following Creative Commons Licence conditions.

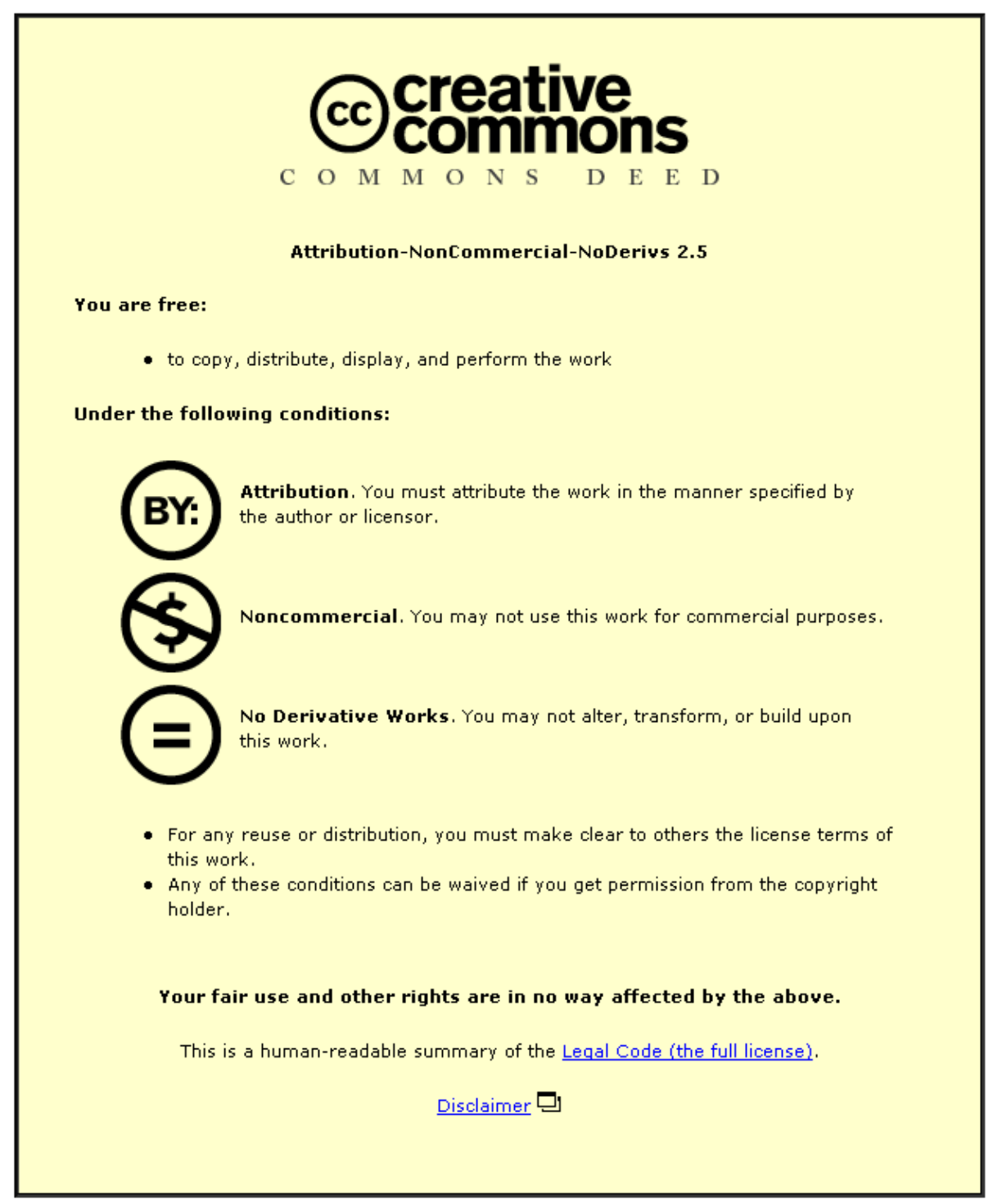

For the full text of this licence, please go to: http://creativecommons.org/licenses/by-nc-nd/2.5/ 


\title{
Developing Incident Causation Constructs for Managing Safety in Construction
}

\author{
YOUISEF AL-SHEHRI ${ }^{1}$ and FRANCIS EDUM-FOTWE ${ }^{2}$ and ANDREW PRICE ${ }^{3}$
}

School of Civil and Building Engineering, Loughborough University, Loughborough, UK.

${ }^{1}$ E-mail: yousef-uk@hotmail.com

${ }^{2}$ E-mail: F.T.Edum-fotwe@lboro.ac.uk

The UK construction industry has a relatively high level of fatal and non-fatal accident rate, which creates the need for improving its safety performance (HSE, 2006). However, the safety performance of the UK is no worse than that of other leading advanced economies, and it may be inferred that improving safety performance is a problem that is shared wider than the UK. While progressive efforts have been directed at improving safety in construction, the level of performance appears to stagnated over the last decade HSE, 2006). The levelling in safety performance would suggest that improving safety further beyond current attainment calls for a radical look at how safety is addressed by construction. Such a radical look would not only cover the planning, implementation, and management of safety in construction, but also give attention to aspects beyond the traditional practices of safety management. Because the incident research was limited this paper focused on the effort of safety management needs to shift from managing and monitoring accidents to managing and monitoring incidents. This will call for a greater understanding of incident causation in construction, and also to divert attention from monitor and manage for accident to monitor and manage for the incident, which mean the incident investigation has two main parts managing (before the incident happened based on analysis the causes of incident to reduce it and reduce accident events which also improve the safety) and monitoring (after incident happened as part of control and management to improve safety in construction) and to develop a system for analysis and then documented on the form of a report and finally deal with the perpetrators of the violation of safety regulations, in order not to repeat it.

Keywords: Incident, Incident Causation, safety culture, Incident management, safety performance.

\section{Introduction}

Construction is often considered as having very unsafe operations compared with other industries. The conditions of work could change on a daily basis and often these present several challenges to the workforce. In addition, construction activity on site is considered as inherently dangerous as well as involving high levels of risk (Hinze and Olbina, 2008). In many national industries across the globe, construction often reflects high rates of accidents. Hinze (2005) for instance argued that the only other industry in the world which has a worse accident record compared to construction is the mining industry. Within the industry, policy makers are aware of the unenviable and unfortunate reputation related to the high level of accidents and death cases, and there is no shortage of initiatives to improve the situation Huang and Hinze (2006). However, establishments such as the UK Health and Safety Executive clearly recognize the huge 
challenge involved in maintaining a health and safety regime for most construction activities (HSE, 2006). Notwithstanding the efforts to reduce construction accidents, injuries and fatalities, the level of these adverse events remain relatively high (Ferjencik, 2011; Smallwood and Haupt, 2005).

The concept of an accident has been defined by the Health and safety Executive in the UK as the unwanted or undesired situation which leads to injury or ill health (HSE, 2006). It further provides that an incident, which is the focus of the research that underpins this paper, is an unwanted or undesired situation, event or episode, which can potentially lead to an accident. The close relationship between the two concepts can be represented as a finely balanced system as shown in figure1. Untreated incident situations often tip the balance towards more accidents. A proactive treatment of incident causal factors prevents such a tip.

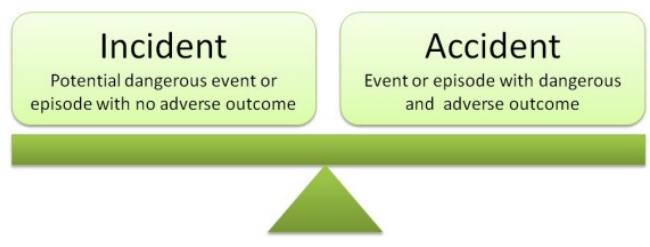

Figure1.The incident - accident balance

There is a wide agreement on the effect that incidents mitigation have on construction safety performance. Notwithstanding such awareness, a relatively limited research attention is given to preventive solutions that take advantage of incidents, as a mechanism for improving safety in construction. Within this paper, the authors focus on the need to shift safety management from managing and monitoring accidents to managing and monitoring incidents. This will calls for a greater understanding of incident causation in construction industry. The paper presents a review of relevant incident related studies and proposes a new concept for more proactive incident mitigation by construction.

\section{Characterizing incident}

\subsection{Incident investigation and reporting}

The importance of incident investigation and reporting system is a main resource that can help construction to determine the safety problems clear and strategic manner. Within construction, incidents are often generated due to variable and unsafe conditions that arise from the operation of a complex delivery system on production site. Most unsafe conditions are caused by influences such as organizational factors, lack of familiarity with adopted technological solutions, as well as method and process related hazards. Cooke and Rohleder (2006) have argued that the adoption of an appropriate reporting system can improve and minimize the level of such unsafe conditions. They further suggest that monitoring required for such reporting often lead to corrective actions arising from incident investigations that contribute to improving safety. Figure 2 provides the conceptual form of reporting that was proposed by Cooke and Rohleder (2006).

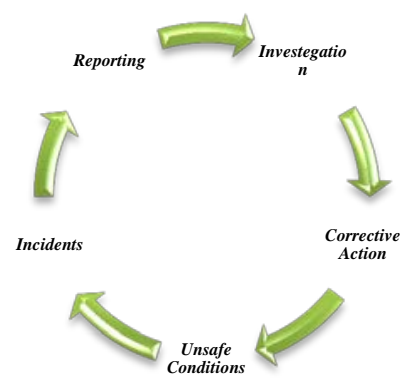

Figure 2. Incident investigations and reporting (Cooke and Rohleder, 2006)

In general it can be argued that unsafe conditions in the work environment and riskprone behavior by the workforce are the main factors that give rise to incidents. Cooke and 
Rohleder (2006) suggest that safety can be improved by reducing these two main incident causal factors. They also argue that the ability and willingness of labor to report incidents is influenced by their general commitment to safety and the effectiveness of the reporting system to proactively raise attention of any potential incidents. Where such a commitment is low, and coupled with an ineffective reporting system, very few potential incident situations will be captured.

At the same time, correcting potential incident situations will be driven by management commitment to safety. Such a management commitment will determine the extent to which episodes are investigated, which in turn will be affected by the resource levels available for episodic mitigation. According to Cooke and Rohleder (2006) the resource level must cater for the study of any reported episode along with its action plan to eliminate its potential future occurrence.

The incident investigation covers two main phases:

- Monitoring (which involves focusing on pre-incident conditions as part of the control and management effort to achieve safety assurance in construction); and

- Managing (which addresses the postincident situation and is often based on analysis of the causes of the incident in order to mitigate future incidents as well as reduce accident events. Figure 3 shows how these two phases give rise to three principal activities for the investigation as advocated by Cooke and Rohleder (2006).

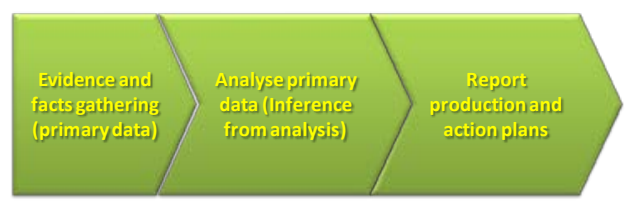

Figure3. Key areas in incident investigation

The role and context of the investigation can best be appreciated by reflecting the concept through a forensics lenses. The notion of forensics involves the application of a broad spectrum of analytical tools to resolve problems and questions that have to a legal interest bearing. The forensics of safety events suggests that within construction each unsafe event can be structured into four key phases as follows:

- Pre-existing conditions;

- Trigger phase;

- Episodic phase; and,

- Adverse impact phase.

The pre-existing conditions map on to the monitoring phase of incident investigation. The trigger phase represents a combination of various factors that initiate the unsafe episode.

\subsection{Analysis of Causes}

The following figure 4 shows the meetings of the whole factors that affect the construction procedure these factors are shaping factors that separated to be material, work team, equipment and work place factors (Reason, 1997). As the figure studied the following can be concluded; the Workplace factors depend on the Site constraints, work scheduling and the housekeeping, while the Work team factors depends on the Attitudes/motivations, knowledge and skills, supervision and the health or the fatigue.

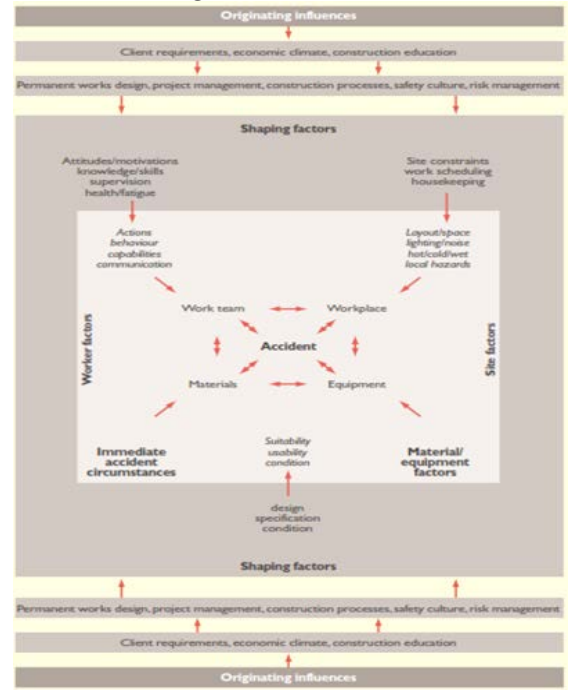

Figure 4. Reason’s model plates (Gibb et al., 2009) 
The Construction Accident Causality (ConCA) established that in order to reach the continual protection from the accidents a focused work from the whole parts that involved in the protection process (Gibb et al., 2009). The fundamental analysis demonstrated in above show that all the individual action in addition to the site hazard had been examined, it was attractive that many of the construction accident happened "off- task", during preparatory activities or even while the workers were moving around the work place. Adding to that, the frequent appearance of the equipments and materials is considered accident causes, despite that are might considered minor incident.

The malfunction of the equipment and the material design or even their actual condition can participate in the failure. The safety required for handling these equipment and material must been given little concern from their designers (Ferjencik, 2011; Hollnagel, 2009; Leveson, 2003).

\section{Review of Safety Improvement}

Within construction, safety is regulated by a national body in most countries either as a department of the government machinery or as an independent executive arm (Morrison, 2004). Often such safety bodies, along with academic interest groups associated with safety performance, often share the aspiration of no accidents or zero accident for the industry.

In exceptional cases, a no incidents goal in delivering construction projects is pursued. The sub-sections that follow explore some of the goals, developments and initiatives that have driven safety performance in a selection of countries to illustrate the point. They are addressed under the categories of UK and Europe, the USA and Australia, and other economies.

\subsection{Developments in the UK and Europe}

The principal agency responsible for safety within the construction industry in the UK is the Health and Safety Executive (HSE). Similar public agencies with the same level of independence exits across the EU countries. The HSE coordinates both practice and research development in safety performance. Institutions that support and promote the safety agenda of the HSE include the European Construction Institute (ECI) and Engineering Construction Industry Training Board (ECITB). Improvement initiatives championed by these institutions include promoting a safety passport agenda, and using a cross-company taskforce to improve safety performance. Such improvement efforts explore safety across the full delivery of the project. Gibb et al. (2009) reported and in order to achieve an important minimization in the amount of accidents a significant effort should be given to the whole system from the designer to the supervisor and the workers, as well as every other member related to the project. Such a focus is driving an agenda of designing to safety in the same mode as the assurance scheme for quality. Simultaneously, initiatives such as 'zero-harm', Considerate Contractor Scheme, Carbon Footprint measures with neutral being the accepted norm, is fostering a new culture for safety on the construction site. These developments should enable safety improvement to become more tactical in the delivery of projects.

\subsection{Developments in USA}

In the USA, the notion of zero-accident and minimal incident rate has been at the forefront of construction and projects for the past two decades (Hinze and Teizer, 2011). While this aspiration is achieved for many projects, there are still instances of accidents and incidents. The recent Gulf of Mexico accident involving Halliburton and BP presents a typical case. The persistence of adverse safety episode in 
Developing Incident Causation Constructs for Managing Safety in Construction

Al-Shehri, Edum-Fotwe and Price

the world's most advanced construction industry underscores the importance of extending the frontiers of the safety agendas if any further improvement can be achieved. It would appear that such an extension should look beyond the production site. Gambatese et al. (2008) evaluated safety risks that originate from the design process and argued that applying safety management systems should make it easier to mitigate most of them. The researchers studied 224 cases of site accidents and concluded that $71 \%$ of the 224 accidents were related to the planning of safety management. Where such planning is conducted at the design phase, it provides a more strategic orientation for safety. The argument here is that planning for safety management should be an important issue and need to be strategically tackled by construction. In some cases even the planning of safety may not exclude the occurrence of accidents, due to the multifarious influences that play out on the site. In general, there is a widely accepted view among professional engineers in the construction that planning for safety management reduces the possibility of accidents. Gambetese et al. (2009) suggested a strong correlation between construction site safety performance and planning for safety management.

According to data from OSHA the USA had 3500 incidence cases per 100,000 in 2010 or $3.5 \%$ rate, down from $3.6 \%$ in 2009 . The improvement correlates with safety performance in US construction, and confirms the potential for improving safety by focusing on the elimination of incidences. Equally, the percentage reduction from 2009 to 2010 means site incident provides a clear and structured approach to identifying the safety risks.

\subsection{Developments in Australia}

The Australian Safety and Compensation Council (2007) reports on incidence rate (per one thousand person) and the data for 2007 reflected a decreased from thirty (30) in the year 2001-2002 to twenty-six (26) for the year 2005-2006. The improvement of with $13 \%$

over the period is associated the introduction of an effective reporting and investigation system based on recognized applied approaches, models and theories that are known to influence incident and accidents as depicted in the model in Figure 5. The model is seen as providing opportunity for identifying factors that are common across three reporting plates. The plates represent factors as the originating influences, shaping factors and immediate circumstances. The rationale of the three plates is that an accident is seen as inevitable once an activity experiences all three plates of the reporting system. The object of the plates is to filter out those incidents that could give rise to accidents before they transpire (Australian Safety and Compensation Council, 2007).

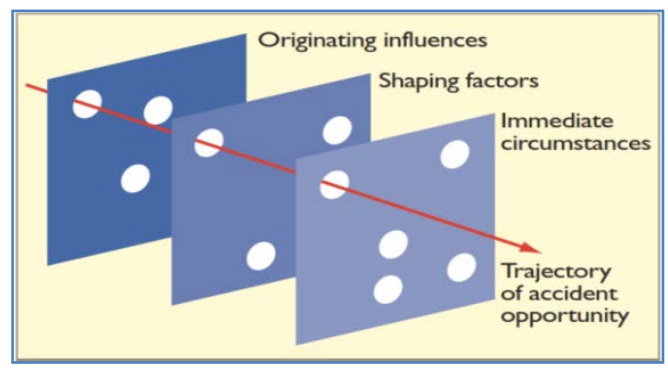

Figure 5. Reason’s model plates (ASCC, 2007).

Additional developments in Australia include the work of Borys (2011), who proposed a Safe Work Method Statements (SWMS) as a system for construction. The SWMS aims to minimize the amount of loses due to accidents by providing a more protected construction environment. The approach adopted in the method statements is to explore all the potential contributory factors that could produce adverse safety performance, and then provide a mitigation plan.

\subsection{Developments from other economies}

While safety improvement issues the three economic regions often focus on how to achieve performance beyond simple conformance, in many other industries across 
the globe, the emphasis of safety is achieving the conformance in the first place (Hon et al., 2010; Chi et al., 2005; Johnson, 2003). Haadir and Panuwatwanich (2008) argue that notwithstanding the volume of construction projects, it is the successful implementation of safety programs that in Saudi Arabia which needs attention. They go on to define seven factors that they deemed critical for successful implementation of safety programs for construction projects in the Saudi industry as follows.

- Suitable supervision

- Safety training

- Effective enforcement

- Teamwork

- Personal attitude

- Clear and reasonable objectives

- Management support

The work of Haadir and Panuwatwanich (2008) clearly recognized two important factors that present an opportunity for taking safety improvement beyond the conformance agenda. The recognition of personal attitude indicates that the individual has a role in the achievement of safety performance, which is often ignored. Similarly, the importance of safety training emphasizes the point about re-conditioning individuals to be more safety conscious and safety oriented.

Current records suggest that in China, the process of construction at the site level is often reflected as a very dangerous process (Zeng et al., 2007; Siu et al., 2004). The authors argue that the high levels of accidents which occur on these sites are due to a number of reasons that relate to the site safety procedures. So a developing new set of site regulations that take into consideration the workers safety was seen as a natural way forward for improving safety. More specifically, they suggested that construction organisation global construction is seen as giving great attention to the safety, especially as defined by Occupational Health and Safety (OHS) Management System and the Occupational Health and Safety Assessment Series OHSAS 18001. They further argued that these two standards are not applied by construction organizations in China. They base their argument on records of accidents for the construction sites over a three (3) year period.

\section{Proposed incident causation study}

The review conducted has established the important role that individuals can have on project safety performance. At the same time, the role of training to re-condition workers suggests that workers with a tendency to be unsafe can be turned around to improve the overall safety performance of a project. Much of such improvement effort would have to focus on the incident stage of safety management to make it effective. Because incident research for construction is generally limited to factor identification, it formed the basis of the focus of this paper as a starting point. The overall purpose of the study from which this paper was derived is to develop the individual contribution to safety as a means for improving safety performance for construction organisations. This paper presents that conceptual model that was developed to support that study, and focuses on the need to shift of safety management from managing and monitoring accidents to managing and monitoring incidents. This will call for a greater understanding of incident causation in construction, and also to divert attention from monitoring and managing for accidents to monitoring and managing incident.

The conceptual model was derived from extensive review of both academic literature and industry initiatives and improvement to safety undertaken in several countries, and is presented in figure 6. Within the subject area of construction safety a significant number of investigators have attempted to establish the 
Developing Incident Causation Constructs for Managing Safety in Construction Al-Shehri, Edum-Fotwe and Price

exact factors and their magnitude, as well as

their nature that give rise to accidents

causation (Hollnagel, 2009). 


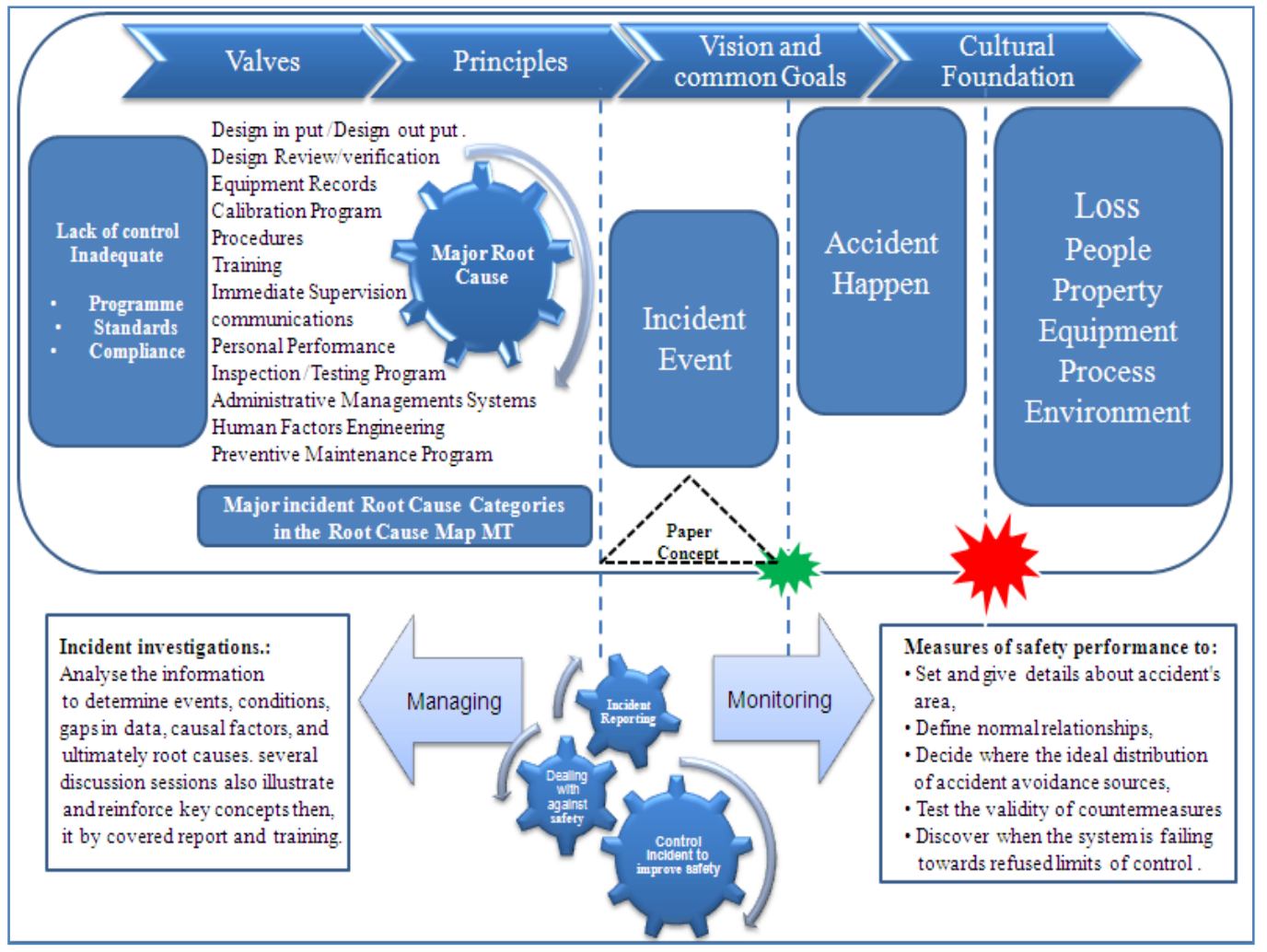

Figure 6. Proposed model to explore incident causation

The incident investigation proposed in the conceptual model has two main parts monitoring and managing. As see in the proposed model figure 6 , in developing conceptual model for incident investigation, the authors relied on extensive research material such as standards, safety systems, as well as tolls and mechanisms for process analysis. This helped to establish the preliminary path for the occurrence of any incident related to construction sites. The proposed model starts from left to right as follows:

Lack of control and Safety inadequacy often reflects a function of three principal parameters. These can be grouped under the following categories.
- Program

- Standards

- Compliance

And based on it, the root cause map will be papered which leads to the identification of the emergence of, and causes of incidents at various levels within the project organisation. The following areas would be explored:

- Design in put /Design out put

- Design Review/verification

- Equipment Records

- Calibration Program

- Procedures

- Training

- Immediate Supervision 
Developing Incident Causation Constructs for Managing Safety in Construction

Al-Shehri, Edum-Fotwe and Price

- Communications

- Personal Performance

- Inspection /Testing Program

- Administrative Managements Systems

- Human Factors Engineering

Specific aspects such as Preventive

Maintenance Program incident investigation has two main parts.

1. Managing pre-incident phase based on analysis of the causes of incident to establish and reduce events which are accident prone.

2. Monitoring phase, which, focuses on post-incident environment and condition as part of control and management to improve safety in construction.

The process can summarized as follows:

\section{Part One: Measures of safety performance to:}

- $\quad$ Set and give details about accident's area,

- Define normal relationships,

- Decide where the ideal distribution of accident avoidance sources,

- Test the validity of countermeasure

- Discover when the system is failing towards refused limits of control.

\section{Part Two: Incident investigations:}

Analyze the information to determine events, conditions, gaps in data, causal factors, and ultimately root causes. Several discussion sessions also illustrate and reinforce key concepts then, it by covered report and training. So need to the training program based on real accidents, to will be successful and comes with the wanted results. The process of developing focused on the following:

- Defining the reason of with a small test plus number of samples for accidents that studied and evaluated so that the trainee will get the picture of the accident process.

- The defining of the managing organization.

- Defining the main reasons, plus a test enhanced in order to sustain the trainee to get them by themselves.

- Define the way that utilized in order to collect the information to study the accidents.

- Define the Causal Factors Charting; in this part the trainee will learn how to make the required in the study at the right time.

- Define the essential reason examination for the accidents; this part aims to teach the trainee the way of using the Root Cause Map during the examination.

- Ask the trainee the best thing or part in the training and the worst in order to avoid the worst and concentrate on the best.

- Define the way to collect the whole information that found out by the examination via the trainee.

\section{Discussion}

The importance of research on the incident that when study it, can be understand the reasons and analysis, and therefore reduce the occurrence of the incident and accident, which has a positive impact on the construction industry and its growth as well as the financial benefits of the project. Productivity is represented by achieving the organization goals, so if the organization is not achieving its goal, pressure is increased to get the required productivity. Losses due to safety incidents will cause a loss of productivity capacity which then increases productivity 
pressure. So, if the business goals didn't be achieved, then the management will tolerate with safety goals.

So incident investigation means searching beyond the obvious or the immediate reason to establish the root causes of accidents. In summary, the study is directed at gathering specific, rich, detailed data about incident factors from causation conditions that relate to selected samples of accidents should lead to a more proactive approach for safety management for the construction sector.

Also the training and learning parts are the most important parts in the process of risk controlling, the safety supervisors should pay attention for these two parts and try to educate the workers and train them to deal with the heavy apparatus and the dangerous materials, and also educate and train the engineers in the parts that related to design for control the risk. The safety management systems are very significant for the sites safety, but unless the safety becomes a culture in the working sites the wanted goals will not be achieved.

\section{Conclusion}

The construction organisation is considered as one of the four most dangerous industries, since this job requires the use of heavy and dangerous equipment which increase the risk at the site level. Equally, the leveling of safety performance improvement presents a matter of concern and requires research attention to ensure a safe working environment in construction. Such a safe working environment can be achieved by focusing attention on incidents as a means to stem accidents. Within this paper, the authors have proposed a conceptual model for conducting such a study, with a view to develop application tools from the results of the investigations. The resulting tools would present a novel option for improving construction safety can be used in improving safety performance by construction organisation.

\section{References}

Borys, D., 2012. "The role of safe work method statements in the Australian construction industry" Safety Science 50, pp. 210-220.

Chi, C.-F., Chang, T.-C., Ting, H.-I., 2005. "Fatal occupational falls in the construction industry in Taiwan”, 1994-1997. Appl. Ergonomics, pp.

Choudhry, R. M., Fang, D., Mohamed, S., 2007. "The nature of safety culture: A survey of the state-of-the-art." Safety Science 45, pp. 993-1012.

Cooke D.L. and Rohleder, T. R., 2006. "Learning from incidents: from normal accidents to high reliability." System Dynamics Review Vol. 22, No. 3, pp. 213-239. Ferjencik, M., 2011, "An integrated approach to the analysis of incident causes”, Safety Science 49 pp. 886-905.

Gambatese, J.A., Behm, M., and Rajendran, S., 2008.

"Design's role in construction accident causality and prevention: Perspectives from an expert panel" Safety Science 46, pp. 675-691.

Gibb, A. Haslam, R. Gyi, D. Hide, S. Duff, R. 2009. What causes accidents. by the author and is made available under the following Creative Commons Licence conditions.

Haadira, Al S. and Panuwatwanich, K., "Critical Success Factors for safety program implementation among construction companies in Saudi Arabia.” Procedia Engineering, The Proceedings of the Twelfth East AsiaPacific Conference on Structural Engineering and Construction - EASEC12, 14, 2011, pp. 148-155 Health and Safety Executive. 2006. Health and Safety in Construction. UK.

Hinze WJ , Olbina S., 2008. "Problem areas in persona; fall protection”. In: Hinze J, Bohner S , Lew J (eds). Proceedings of CIB W99, $14^{\text {th }}$ Rinker International Conference, Gainesville, Florida, March 2008:406-18 Hinze, J.W. and Teizer, J., 2011 "Visibility-related fatalities related to construction equipment.” Safety Science 49, pp. 709-718.

Hinze, J.W., 2005. A paradigm shift: leading to safety. In: Proceedings of the CIB W 99, 4th Triennial International Conference: Rethinking and Revitalizing Construction Safety, Health, Environment and Quality, 17-20 May, Port Elizabeth, South Africa, pp. 01-11.

Hollnagel, E., 2009. "How accident investigations contribute to learning: what you look for is what you 
Developing Incident Causation Constructs for Managing Safety in Construction Al-Shehri, Edum-Fotwe and Price

find.” In: JRC/ESReDA Seminar on Lessons Learned from Accident Investigation, Coimbra, Portugal, June pp. 2-3.

Hon, C.K.H., Chan, A. P.C. and Wong. F.K.W., 2010. "An analysis for the causes of accidents of repair, maintenance, alteration and addition works in Hong Kong” Safety Science 48, pp. 894-901.

Huang, X. and Hinze, J., 2006. “Owner's role in construction safety.” Journal of Construction Engineering and Management. 132 (2), pp. 164-173.

Johnson, C.W., 2003. Failure in Safety-critical Systems: A Handbook of Incident and Accident Reporting. Glasgow University, PressGlasgow. ISBN:0-85261-784-4.

Leveson, N.G., 2003. “A new model for analyzing accidents”. In: JRC/ESReDA, Seminar on Safety Investigation of Accidents, Petten, The Netherlands, 12-13 May 2003.

Morrison, L.M., 2004. "Best practices in incident investigation in the chemical process industries with examples from the industry sector and specifically from Nova Chemicals”. Journal of Hazardous Materials 111, pp. 161-166.

Reason, J.T. 1997., Managing the Risks of Organizational Accidents, Ashgate, Aldershot.

Siu, O., Phillips, D.R., and Leung, T., 2004. "Safety climate and safety performance among construction workers in Hong Kong The role of psychological strains as mediators” Accident Analysis and Prevention 36, pp. 359-366.

Smallwood, J. and Haupt, T.C. 2005. "The need for construction health and safety and the construction regulations: engineers' perceptions.” Journal of the SouthAfrican Institution of Civil Engineering, vol. 47, no. 2, pp

Zeng, S. X., Tam, C. M., Deng, Z. M. and Tam, Vivian W. Y., 2003. "ISO 14000 and the construction industry: Survey in China.” Journal of Management in Engineering, ASCE, 19(3), pp. 107-115 